\title{
Directional Communication System for Short-Range Vehicular Communications*
}

\author{
T.D.C. Little, A. Agarwal, J. Chau, M. Figueroa, A. Ganick, \\ J. Lobo, T. Rich, P. Schimitsch \\ Department of Electrical and Computer Engineering \\ Boston University, 8 Saint Mary's St., Boston, MA 02215 USA \\ (617) 353-9877 \\ tdcl@bu.edu
}

MCL Technical Report No. 05-10-2010

May 10, 2010

\begin{abstract}
Improving safety in vehicles is achieved today by improving their ability to perceive and react to threats in the environment. Much effort is directed towards increasing the safety envelope around each vehicle with the introduction of increasingly sophisticated sensor technology (e.g., ultrasound, video, thermal imaging, and LIDAR). This envelope can also be increased by leveraging the exchange of safety messages between neighboring vehicles through localized communications between and among vehicles. This paper describes the requirements for messaging in a nearest-neighbor data interchange for several types of safety threats and makes a case for directional communications. The results of our analysis indicate performance limitations for omnidirectional communications and favors a directional scheme. The packet delay approaches 300ms for IEEE 802.11 at a density of 100 vehicles $/ \mathrm{km}$ in saturation mode for backoff window parameter $(\mathrm{W}=128)$. This value is equivalent or close to the human reaction time and is considered unsuitable for safety messaging applications. The design and development of a prototype directional communication system implemented with optical transceivers is described; its related application in providing improved situational awareness in conjunction with an in-car computer platform connected to a local GPS unit and on-board data monitoring interface is also described.
\end{abstract}

Keywords: Vehicular safety, safety messaging, directional communications, situational awareness, optical communications, WAVE, DSRC, intelligent transportation systems.

${ }^{*}$ In Proc. 2nd IEEE Vehicular Networking Conf. (VNC 2010), Jersey City, December 15, 2010. This material is based upon work supported by the National Science Foundation under Grant No. EEC-0812056. 


\section{Introduction}

The goal of improving safety in transportation is invariant. Two approaches are emerging to achieve this goal: (1) increasing the safety of individual vehicles through sophisticated sensors and on-board warning systems, and (2) increasing the safety of vehicles through communications with nearby vehicles. The latter can be achieved by enabling vehicles to be interconnected using networking technology. This use of collaborative sensing is a means by which vehicles can share sensed data about a vehicle's surroundings or about its current operating state: position, velocity, acceleration, and other vehicular operating characterizations. Such vehicular networking technology has the potential to introduce profound and transformative advancement in safety, traffic, and convenience [1].

Several technologies for message dissemination in vehicular ad hoc networks (VANETs) have been explored; these include cellular, IEEE 802.11 (Wi-Fi), Ultra-Wide Band (UWB), and Free-Space Optics (FSO) [2, 3]. Each technology has different operating characteristics. Furthermore, applications in VANETs such as safety messaging, traffic, and Internet access, $[1,4]$, each has different requirements for bandwidth, latency and infrastructure. For example, Internet access requires infrastructure such as access points or cellular towers for connectivity to the backbone network. Applications providing information about traffic conditions have relatively relaxed latency constraints and involve collecting information from multiple sources (e.g., vehicles, road-based sensors, and highway cameras); such applications can be instantiated without infrastructure support by using multihop communication and networking.

However, because safety infractions occur due to the physics of moving vehicles relative to each other and other obstacles, there is a time-criticality to the sourcing and utilization of sensory information about a vehicle. Ultimately this information can be used to enhance or out-perform the human operator(s) of vehicles but it is perishable, becoming quickly out of date and irrelevant to the current state of the vehicle and its environment. As a result, we anticipate that data exchange will be frequent with small payloads sufficient to capture current and predicted state. Due to the potential for damage in case of failure, safety applications can also require highly reliable message delivery, which can be quantified through the packet delivery ratio (PDR) and the average delay of messages delivered.

To support these varied requirements, complementary technologies must coexist to serve the connectivity requirements of vehicular environments, much like Bluetooth and Wi-Fi do for personal computers. In this paper we compare two groups of technologies: omnidirectional IEEE 802.11 and directional free-space optical communications (FSO).

The Dedicated Short Range Communication (DSRC) spectrum [5], has been allocated for development of the IEEE 802.11p standard for inter-vehicle communication. The draft is a modification of the IEEE 802.11a standard that employs the use of DCF (Distributed Coordination Function). The implementation uses broadcasts to enable vehicles to share state information in a fast and efficient manner with minimal setup time. However, this broadcast methodology creates contention for the wireless medium in scenarios in which the vehicular traffic density is high. When coupled with the potentially high frequency of data 


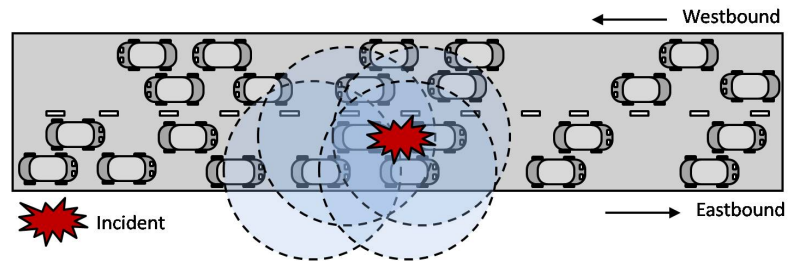

Figure 1: Figure illustrating the 'Broadcast Storm' problem. An incident occurring on a dense highway generates many high priority messages between vehicles leading to contention in the network.

exchange, this can create a 'Broadcast Storm'scenario, in which several nodes broadcasting at a high frequency can effectively jam the wireless space [6]. This is illustrated in Fig. 1 which shows how an incident on a highway can trigger safety communication between vehicles, thereby creating a broadcast storm. Contention in these scenarios increases both latency and the number of dropped packets. This contradicts goals for safety-critical communication systems [7]. A related work aims to develop high-frequency radio techniques that are directional [8]. Such directional communication technologies are advantageous since the directionality limits contention.

In this paper, we describe the basis for, and the design of, a prototype device for exchanging vehicle state among neighboring vehicles. The device uses optical signals to provide directional and line-of-sight communication for vehicle-to-vehicle (V2V) communications and safety messaging with other units (Fig. 2). We also explore the performance of FSO in packet delivery, average delay, and throughput, especially in the context of safety applications in high vehicular density scenarios.

The remainder of this paper is organized as follows: Section 2 presents background information on safety messaging for vehicular networks and directional communication technologies. Sec. 3 compares directional communications with omnidirectional communications to show our motivation for the development of our prototype system. The transceiver design (Sec. 4.1), the software requirements (Sec. 4.2), and the implementation testbed currently under development are also described. Sec. 5 concludes the paper with a summary and directions of future research.

\section{Related Work}

The DARPA Grand Challenge [9] seeks to develop technologies to enable vehicles to use actuators and sensors to drive autonomously. Google engineers have a similar vision for the future of driving [10]. However, these systems heavily depend on on-board sensors and do not exploit the potential of fleets of vehicles supporting inter-vehicle data exchange. Our proposed system seeks to create a communication framework that will expand the vehicle's situational awareness and will lead to automatically-driving vehicles that coordinate with one another. Researchers at USP [11], who are working to develop mathematical models for 


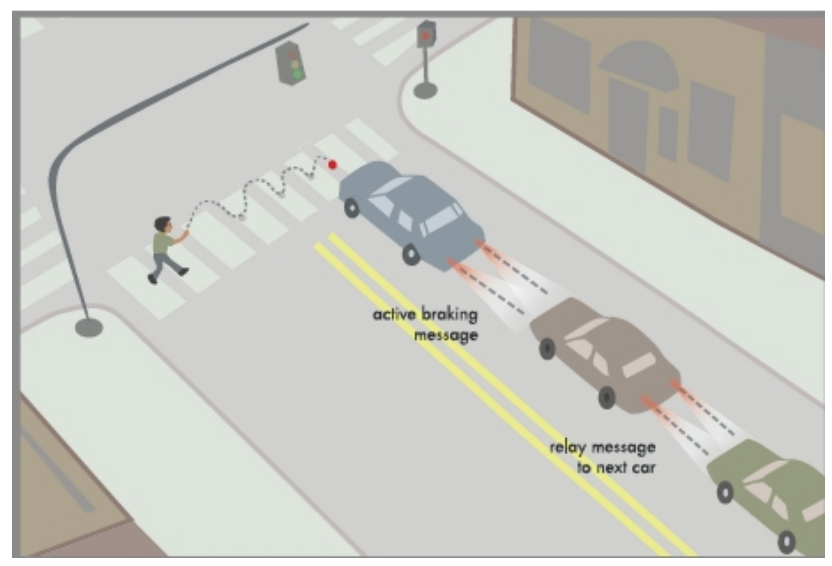

Figure 2: An example scenario where free-space optical communications can be used for safety messaging is depicted. When the front-most car suddenly brakes, it uses its taillights as a transmitter to notify the car immediately behind it. This car in the middle can then relay this message to the next car to prevent a rear-ending by the next car.

autonomous driving, have expressed the importance on the role of communication in future vehicles.

Several works have considered the use of FSO for vehicular networking. Arai et al. have created a data dissemination system based on LED traffic lights; it will modulate light from LEDs at high rates to transfer data to vehicles that have receivers in the form of high speed cameras [3]. Intel has demonstrated an active-braking application using LED-based communication between vehicles [12]. Our group at Boston University is currently working to develop optical transceivers that can be installed on vehicles an enable directional and line-of-sight vehicle-to-vehicle communication.

DSRC or Dedicated Short Range Communication is a band of frequencies at $5.9 \mathrm{GHz}$ allocated for WAVE (Wireless Access in Vehicular Environments) [5]. The IEEE 802.11p task-group is a drafting standards and protocols for communication between vehicles and road-side infrastructure such as access points [7]. Several works have created models for IEEE 802.11 that match real-world observation very closely. In particular we refer to the work by Bianchi [13] in developing a Markov model for the IEEE 802.11 DCF (Distributed Coordination Function). Authors in reference $[14,15,16]$ have modeled the steady-state performance of IEEE 802.11 under saturated and unsaturated conditions. Authors in [6], have extensively studied the broadcast storm problem in an implementation using IEEE 802.11 and suggested modifications to the draft.

We base our analysis on these prior works with the additional constraints imposed by optical communications but otherwise corresponding performance characteristics. Part of our goal is to develop a system that can match or exceed these performance parameters given the physical constraints from line-of-sight communications.

In the context of mathematical modeling, we rely on works that have been shown to be in strong agreement with real-world observations $[13,14]$. We extend these models to 
study vehicular networking scenarios with high-density bidirectional vehicular traffic and high packet rates. We are primarily concerned with contention and focus on the analytical results based on these models. We assume that nodes are exponentially distributed on the roadway, which is consistent with related work [17]. We concentrate our analysis on scenarios where the density distribution of vehicles ranges from a sparse density of 10 vehicles $/ \mathrm{km}$ to a concentrated density of 100 vehicles $/ \mathrm{km}$. Given the high mobility of vehicles in the system and the relatively random arrivals and departures of nodes in the system, we seek to minimize the connection establishment time by assuming that vehicles exchange state information via broadcasts. We assume that vehicles are constantly exchanging state information. As a result there is a high frequency of data exchange, and thus, even with a small number of nodes in the system, there is a state of saturation. This assumption is distinct from related works that do not always assume a high data-rate.

Figure 3(a) shows a car computer integrated with an optical transceiver. The car computer is an off-the-shelf component that is equipped with a touch-sensitive screen, a fan-less CPU for operation in harsh environments, and wireless communication capabilities [18]. It has eight USB ports for connecting to various devices. Our optical transceivers are connected to this device to enable the car computer to exchange information with other vehicles in the system.

Figure 3(b) shows the optical transceiver used in this system. Each transceiver consists of a narrow-beam white LED-based transmitter and a photodiode-based receiver. The LEDs are used to modulate light at a rate above what the human eye can perceive. The narrowbeam LEDs on the transmitter and the lens in front of the receiver improve signal gain and directionality. The transceiver's design was adapted from designs originally developed for indoor use, where visible-light communication systems, when used in lieu of regular lighting or radio communication systems, can yield many potential benefits; these benefits include reduced radio-frequency interference, greater throughput per unit area, and improved energy efficiency.

\section{Directional Vs. Omnidirectional Communication}

We investigated the tradeoffs in the adoption of IEEE 802.11 and free-space optical communication (FSO) for vehicular communication applications. We used a model provided by Ma and Chen [14] for an IEEE 802.11 broadcast mechanism operating in the saturation state to compute the packet delivery ratio, packet delay, and throughput for various node densities in the network. This model was also applied to the FSO case for comparison. The IEEE 802.11 radio standard is assumed to have a range of $200 \mathrm{~m}$ while FSO is expected to have a communication range of $50 \mathrm{~m}$. We also assumed that the wireless radio is omnidirectional while FSO is directional. Their behaviors under these assumptions were compared to determine the influence of various parameters on the performance. Traffic density was varied from a low of 10 vehicles $/ \mathrm{km}$ to a high of 100 vehicles $/ \mathrm{km}$. An exponential distribution of nodes on a liner roadway was assumed, which is consistent with prior work 
[17]. The simulation parameters for IEEE 802.11 and FSO modeling are given in Table 1.

Table 1: Parameters used for IEEE 802.11 and FSO modeling

\begin{tabular}{|l|l|}
\hline Parameter & Value \\
\hline Packet Payload, $P$ & 8184 bits \\
\hline MAC Header, $M A C$ & $272 \mathrm{bits}$ \\
\hline PHY Header, $P H Y$ & $128 \mathrm{bits}$ \\
\hline Channel bit rate, $R$ & $1 \mathrm{Mbit} / \mathrm{s}$ \\
\hline Propagation Delay, $\delta$ & $1 \mu \mathrm{s}$ \\
\hline DIFS & $128 \mu \mathrm{s}$ \\
\hline Slot time $\tau$ & $50 \mu \mathrm{s}$ \\
\hline Backoff window size, $W$ & $32,64,128$ \\
\hline
\end{tabular}

Figure 4(a) shows a comparison of the number of nodes in the neighborhood (or within the communication range) of a vehicle that is equipped with IEEE 802.11 or FSO technology. In a bidirectional highway scenario with numerically equal traffic densities in both directions, there are potentially 80 vehicles within the neighborhood of a vehicle using IEEE 802.11. However, with the shorter-range FSO technology, its $50 \mathrm{~m}$ range limits reception of data from vehicles that are beyond a vehicle's immediate neighborhood; even at the extreme density of 100 vehicles $/ \mathrm{km}$, the average number of nodes within the neighborhood of a single FSO receiver is 10 . With fewer nodes competing to transmit on the wireless medium, contention is reduced. Thus, with respect to contention, directional and short-range wireless techniques are advantageous, especially in high density scenarios.

Figure 4(b) shows a the packet delivery ratios (PDR) as a function of the number of nodes in the network. For IEEE 802.11, the PDR rapidly decreases as the number of nodes increase due to increased contention; on the other hand, the FSO technique has limited contention due to the directionality and limited range. As the backoff window $(W)$ is increased, the packet delivery ratio becomes higher. However, even with an increased backoff window size $W$, the packet delivery ratio for IEEE 802.11 falls below $50 \%$. For the FSO implementation, the packet delivery rate is higher and only falls below $80 \%$ in very high-density scenarios.

In Figure 5(a), the average packet delay in data exchange is shown. For the IEEE 802.11 technique, packet delay is at least 0.05 seconds in low density scenarios and reaches 0.3 seconds in high density scenarios. The packet delay increases because the increased contention in the medium causes collisions and backoff, which forces the nodes to wait for a longer time. Increasing the backoff window size increases the delay since the nodes have to wait for more time slots. For FSO, the delay is always below 0.05 seconds.

Related work has established that packet delay in vehicular communication for safety applications must be strictly below $400 \mathrm{~ms}$ [7]. While the scenarios shown here meet this 
criteria, other scenarios exist where this criteria is not achievable. Thus, backoff window size $(W)$ is an important design consideration in protocols for safety communication.

In Figure 5(b), the normalized throughput is shown. For IEEE 802.11, the results show a trend similar to that of the packet delivery ratio. Note that as the collisions increase, both the packet delivery ratio and the channel utilization decrease. By increasing the backoff window size, packet collisions can be prevented, thereby increasing channel utilization. Also note that for FSO, for $W=32$, the channel utilization rate actually increases with the vehicle density. This phenomenon is due to how the nodes wait in backoff mode when the number of nodes competing for the channel are low. In FSO, the drop in throughput is not as severe as that in IEEE 802.11.

The analytical model has been used to show the potential trade-offs between using IEEE 802.11 radios and using short-range, directional communication implemented through freespace optical communications. Applications such as safety and emergency messaging require very high reliability. However, due to the shared medium access and application of broadcast mechanisms for wireless communication, the probability of a successful transmission decreases exponentially with increasing node density in the network. This phenomenon is known as the broadcast storm problem in ad hoc networks.

This phenomenon exists for both the IEEE 802.11 and FSO technologies. However, since FSO is short-range and directional, the interference due to a high density of nodes in the network is less than that experienced by the omnidirectional wireless IEEE 802.11. As observed from the results, the average packet delay is approximately $0.3 \mathrm{~s}$ for highdensity scenarios, which may be unsuitable for data delivery constraints in design of safety applications in vehicular networks. Furthermore, parameters such as backoff window size were studied to determine the packet delivery ratio, delay, and throughput achieved. Note the trade-off between delivery ratio and delay: by increasing the backoff window, collisions can be avoided, however, the delay in packet delivery is also increased. Thus, the backoff window size is an important design consideration for any safety protocol for vehicular communication, where the communication subsystem must guarantee packet delivery within delay limits.

\section{Prototype System}

\subsection{System Design}

To apply and to experimentally verify of the results of the analysis, a prototype (Fig. 6) was developed to exchange state information as cars would. As advised by the analysis, in order to reduce contention, which may make the system unsuitable for safety messaging applications, the system was equipped with short-range and directional optical transceivers.

Although FSO's short-range, line-of-sight, and directional nature helps to alleviate contention in high-density $\mathrm{V} 2 \mathrm{~V}$ networks by restricting communications to the field of view, this property 
also has some drawbacks. One such problem is depicted in Fig. 7, where vehicle A cannot directly communicate with vehicle $\mathrm{D}$. The highly directional nature also hampers direct communications with cars located on a diagonal.

To overcome these problems, multihop networking was used to allow communications in these situations. This scheme is also depicted in Fig. 7, where car A can communicate with car D through intermediaries B and C. Furthermore, in addition to the forward and rear-facing transceivers depicted as headlights and taillights in Fig. 7, transceivers facing left and right were added to the prototype to facilitate communications with vehicles in adjacent lanes (Fig. 8).

Internally, the prototype consists of sensing and data-collection devices, a touch-screen as the user-interface, and a computer with specialized software to manage the system. Internal sensors, such as the built-in GPS receiver; and data-collection devices, such as an OnBoard Diagnostics Interface (OBD-II) provide the on-board computer with information to determine the attached vehicle's state.

In turn, as depicted in Fig. 9, the car computer can use this information to provide feedback to the user via the touch-screen or to inform neighboring vehicles via FSO.

\subsection{Software}

The software, which allows the system to provide its intended functionality, is divided into two parts: data management and serial communication. The data management modules handle packet data (Fig. 10), and perform functions such as decoding, encoding, timeout, and data display. The data packets contain fields for vehicle identification numbers, timestamps, and GPS information; additional empty space has been reserved in the packet structure to allow for future application development. The timestamp field indicates the age of the packet and allows the system to discard stale information. The GPS fields, such as longitude, latitude, speed, and bearing, will allow vehicles to determine each other's relative position without the need for additional sensors. The serial communication modules interface directly with the optical transceivers to facilitate data transmission and reception.

The software has five main functions: it creates data packets, transmits data, decodes received data, manages and analyzes received data, and displays relevant information to the driver. Data are stored in a queue which prioritizes information and accordingly times it out when deemed necessary. The software takes the most recent queue data that it has received and retransmits the information along with its current state information to its neighbors.

\subsection{System Operation}

The system prototype is a proof-of-concept for vehicle-to-vehicle optical communication. While safety messaging is a primary objective for this project, such a communication system 
can support a variety of other applications. The nodes (or vehicles) in our current system form an optical multihop network. In this system, the node with four built-in transceivers (Fig. 6) acts as an intermediary node that receives information from neighboring "stand alone" single-transceiver nodes. Each node constantly transmits information regarding its current state while waiting to hear from other neighboring nodes. The information received by each node is then appended to its own broadcast stream in order to inform other neighbors.

The node's current GUI (Graphical User Interface) (Fig. 12) presents the information stored in the node's queues: the node's most recently received information. In the case illustrated by Fig. 12 the node has received information from four other nodes, which is shown in four squares under the "Neighboring Nodes" heading. This information is retransmitted along with the vehicle's own state information, which is shown in the square under the "Root Node" heading.

The next version of the GUI will, in addition to other relevant information, also indicate the location of neighboring vehicles (Fig. 13). Some examples are shown in Fig. 13 for four scenarios: with a vehicle located in (a) the rear, (b) the front, (c) a diagonal, and (d) with vehicles on all sides of the user.

\section{Conclusion and Future Work}

Vehicular networks can serve many purposes ranging from in-car entertainment, Internet access, and navigation; to safety messaging for immediate, and near-term safety threats such as hazards from construction sites. Through analytical models, the performance of both long-range, omnidirectional communication and shorter-range, directional communication technologies were evaluated in the context of safety messaging. Although long-range, omnidirectional communication technologies, such as IEEE 802.11, are able to communicate with more neighboring cars directly, this feature becomes a disadvantage in scenarios with high vehicle densities; the increase in the number of vehicles within range of one another increases contention, which in turn increases packet loss and delay. Furthermore, broadcast storms may exacerbate these problems in scenarios where safety messaging is needed most, such as in the case of a car accident. Since safety-related messaging applications require reliable delivery and low latency, technologies such as IEEE 802.11 are not ideal for such scenarios of safety messaging. However, shorter-range, line-of-sight, directional communication technologies, such as FSO are less susceptible to problems arising from contention. This property allows them to achieve shorter packet delays, lower packet losses, and to avoid the broadcast storm problem. These advantages make FSO more suitable for safety messaging applications in vehicular networks.

The results of this analysis provide the motivation for the design and construction of a prototype FSO-based vehicular communication system. This system serves to inform a user of relevant information about nearby vehicles, serves as a proof-of-concept, and is a foundation for additional efforts in vehicular communications. Our future work includes deployment of the optical transceivers on real vehicles, experimentation, validation of utility 
in providing situational awareness to drivers, and integration into cooperative collision avoidance systems.

\section{References}

[1] T. D. C. Little and A. Agarwal, "Connecting Vehicles to 'The Grid'," in Proc. NITRD National Workshop on High-Confidence Automotive Cyber-Physical Systems, Troy, MI, April 2008.

[2] Y. Zang, S. Sories, G. Gehlen, and B. Walke, "Towards a European Solution for Networked Cars - Integration of Car-to-Car technology into cellular systems for vehicular communication in Europe," Speech, ITU, Geneva, Switzerland, p. 14, Mar 2009. [Online]. Available: http://www.comnets.rwth-aachen.de

[3] S. Arai, S. Mase, T. Yamazato, T. Endo, T. Fujii, M. Tanimoto, K. Kidono, Y. Kimura, and Y. Ninomiya, "Experimental on Hierarchical Transmission Scheme for Visible Light Communication using LED Traffic Light and High-Speed Camera," in IEEE Vehicular Technology Conference (VTC-Fall '07), 2007, pp. 2174-2178.

[4] T. D. C. Little and A. Agarwal, "An Information Propagation Scheme for Vehicular Networks," in Proc. IEEE Intelligent Transportation Systems Conference (ITSC '05), Vienna, Austria, September 2005.

[5] Q. Xu, T. Mak, R. Sengupta, and J. Ko, "Vehicle-to-Vehicle Safety Messaging in DSRC," in Proc. 1st ACM Workshop on Vehicular Ad Hoc Networks, Philadelphia, USA, October 2004, pp. 19-28.

[6] O. Tonguz, N. Wisitpongphan, J. S. Parikh, F. Bai, P. Mudalige, and V. Sadekar, "On the Broadcast Storm Problem in Ad hoc Wireless Networks," oct. 2006, pp. $1-11$.

[7] ASTM E2213-03, "Standard Specification for Telecommunications and Information Exchange Between Roadside and Vehicle Systems - $5 \mathrm{GHz}$ Band Dedicated Short Range Communications (DSRC) Medium Access Control (MAC) and Physical Layer (PHY) Specifications," ASTM Std., Tech. Rep., September 2003.

[8] R. Daniels and R. Heath, "60 GHz Wireless Communications: Emerging Requirements and Design Recommendations," IEEE Vehicular Technology Magazine, vol. 2, no. 3, pp. 41-50, September 2007.

[9] L. Grossman. (2008, March) Building the Best Driverless Robot Car. [Online]. Available: http://www.time.com/time/magazine/article/0,9171,1684543,00.html

[10] J. Ramsey. (2009, July) Google phone designers plot for "the end of driving" with autonomobile. [Online]. Available: http://www.autoblog.com/2009/07/08/ google-phone-designers-plot-for-the-end-of-driving-with-autono/ 
[11] USP researchers say future cars will communicate to avoid collisions. [Online]. Available: http://www.usp.ac.fj/news/story.php?id=416

[12] K. Greene. (2009, June) A vision for headlight communications. [Online]. Available: http://technologyreview.com/

[13] G. Bianchi, "Performance Analysis of the IEEE 802.11 Distributed Coordination Function," IEEE J. Selected Areas in Communications, vol. 18, no. 3, pp. 535-547, Mar 2000.

[14] X. Ma and X. Chen, "Performance Analysis of IEEE 802.11 Broadcast Performance Analysis of IEEE 802.11 Broadcast Scheme in Ad Hoc Wireless LANs," in IEEE Trans. on Vehicular Technology, vol. 57, no. 6, November 2008.

[15] J. An, X. Guo, and Y. Yang, "Analysis of collision probability in vehicular ad hoc networks," in GEC '09: Proc. 1st ACM/SIGEVO Summit on Genetic and Evolutionary Computation. New York, NY, USA: ACM, 2009, pp. 791-794.

[16] V. Gau, C.-W. Huang, and J.-N. Hwang, "Reliable Multimedia Broadcasting over Dense Wireless Ad Hoc Networks," in Special Issue on Multimedia Communications, Networking, and Applications,. J. of Communications, Academic Publisher, October 2009, pp. 614-627.

[17] R. Fracchia and M. Meo, "Analysis and Design of Warning Delivery Service in Intervehicular Networks," IEEE Trans. on Mobile Computing, vol. 7, no. 7, pp. 832-845, 2008.

[18] (2010, March) Micro Intel Vehicle \& Home Media Computer. [Online]. Available: $\quad\left\{\left\{\right.\right.$ http://store.mp3car.com/Ultimate $\backslash \_$Micro $\backslash$ Intel $\backslash$-Vehicle $\backslash$ Computer $\backslash$ -p/ccc-208.htm\}\}

[19] A. Agarwal and T. D. C. Little, "Role of Directional Wireless Communication in Vehicular Networks," in Proc. IEEE Intelligent Vehicles Symposium (IV '10), San Jose, CA, June 2010.

[20] X. Ma, X. Chen, and H. H. Refai, "Performance and Reliability of DSRC Vehicular Safety Communication: a Formal Analysis," EURASIP J. on Wireless Communication Networks, pp. 1-13, 2009.

[21] A. Agarwal, D. Starobinski, and T. D. C. Little, "Analytical Model for Message Propagation in Delay Tolerant Vehicular Ad Hoc Networks," in Vehicular Technology Conference (VTC-Spring '08), Singapore, May 2008, pp. 3067-3071.

[22] S. Ashley, "Crashless cars: Making driving safer," in Scientific American, December 2008.

[23] V. Navda, A. P. Subramanian, K. Dhanasekaran, A. Timm-Giel, and S. Das, "MobiSteer: Using Steerable Beam Directional Antenna for Vehicular Network Access," in Proc. 5th Intl. Conf. on Mobile Systems, Applications and Services (MobiSys '07). New York, NY, USA: ACM, 2007, pp. 192-205. 
[24] A. Agarwal and T. D. C. Little, "Prospects for Networked Vehicles of the Future," in Proc. Smart Transportation Workshop in IEEE Real-Time and Embedded Technology and Applications Symposium (RTAS), Bellevue, WA, USA, April 2007.

[25] J. Mittag, F. Schmidt-Eisenlohr, M. Killat, J. Härri, and H. Hartenstein, "Analysis and Design of Effective and Low-overhead Transmission Power Control for VANETs," in Proc. 5th ACM Intl. Workshop on VehiculAr Inter-NETworking (VANET '08). New York, NY, USA: ACM, 2008, pp. 39-48. 


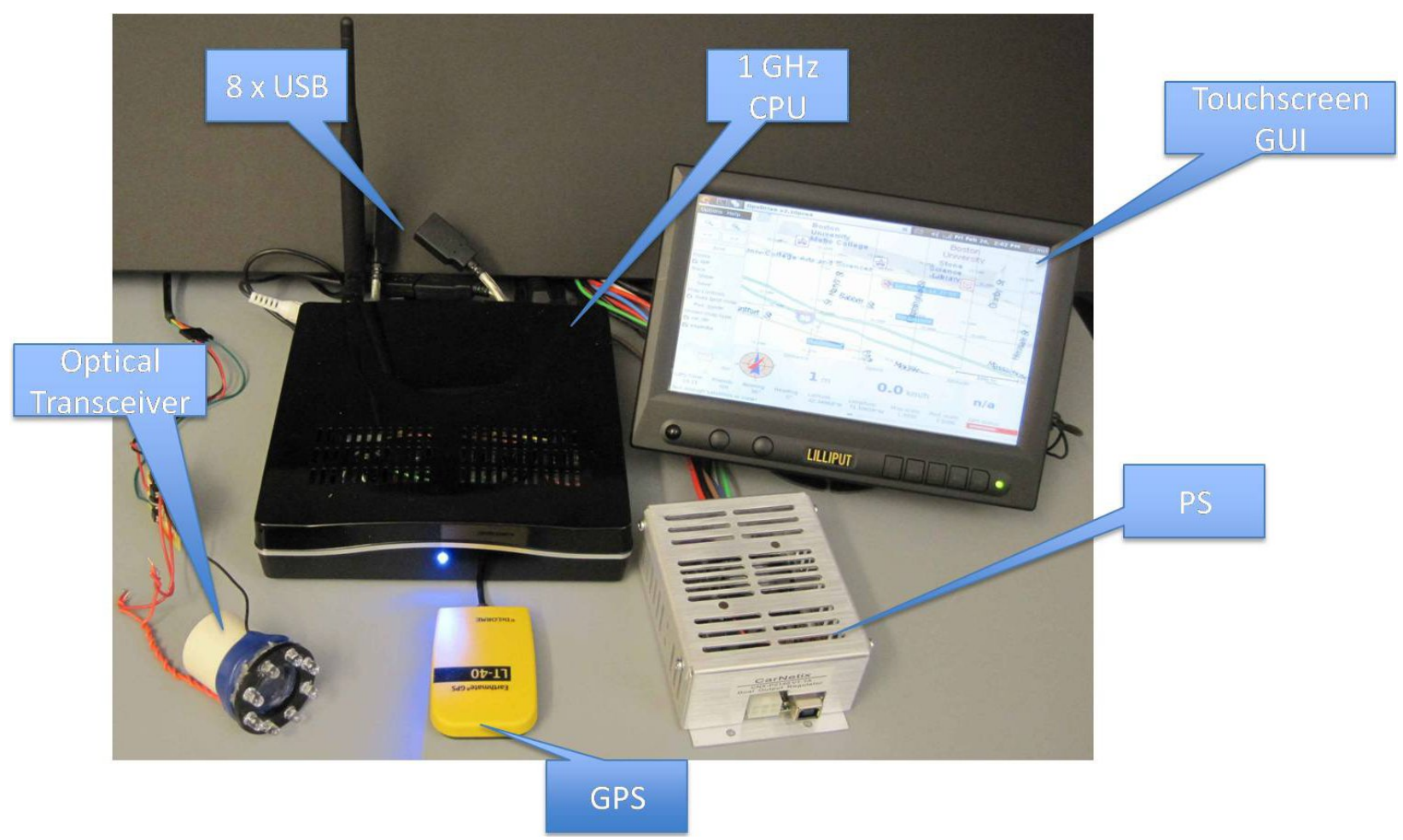

(a)

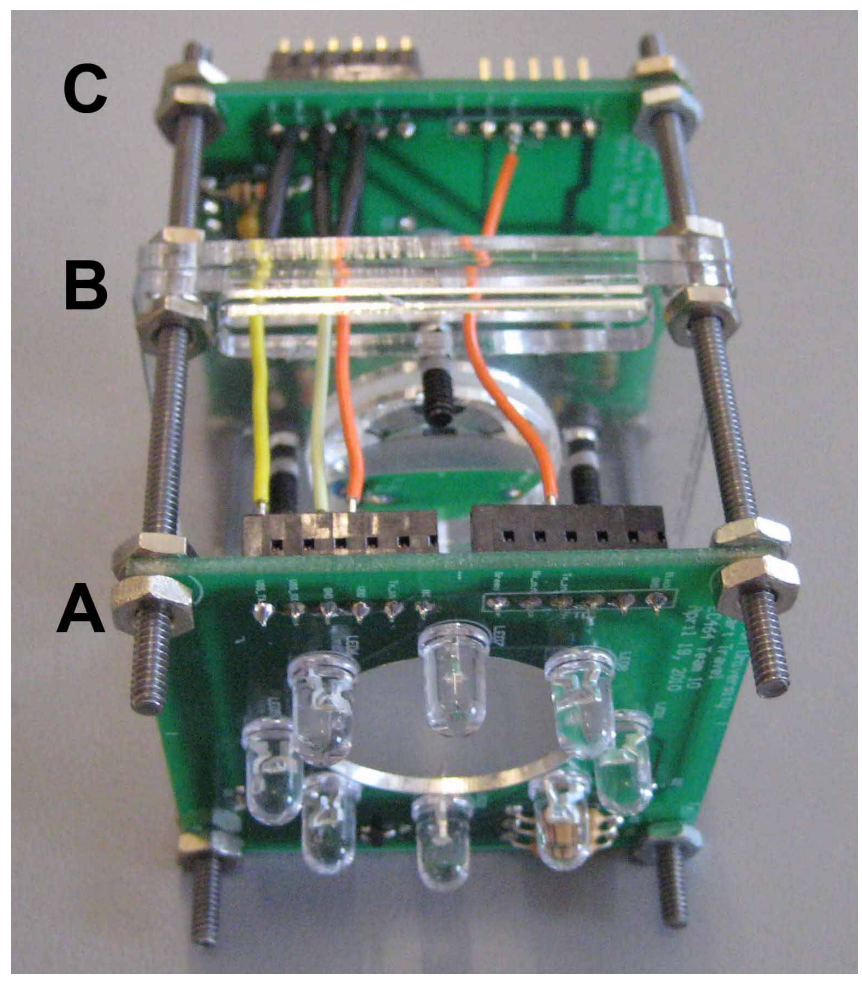

(b)

Figure 3: Photographs of our current prototype. (a) Counter-clockwise from the top right, the touchscreen user interface, car computer, optical transceiver, GPS receiver, and automotive DC power supply. (b) components of the optical transceiver: (A) transmitter, (B) lens, and (C) receiver. 


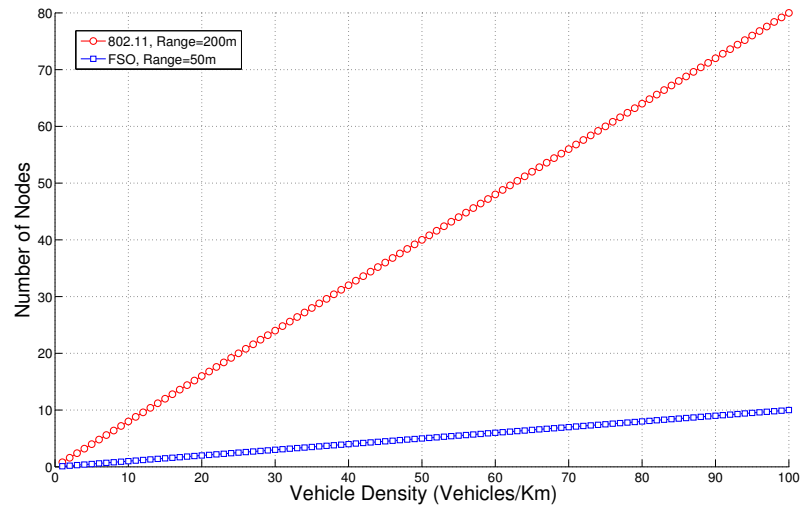

(a)

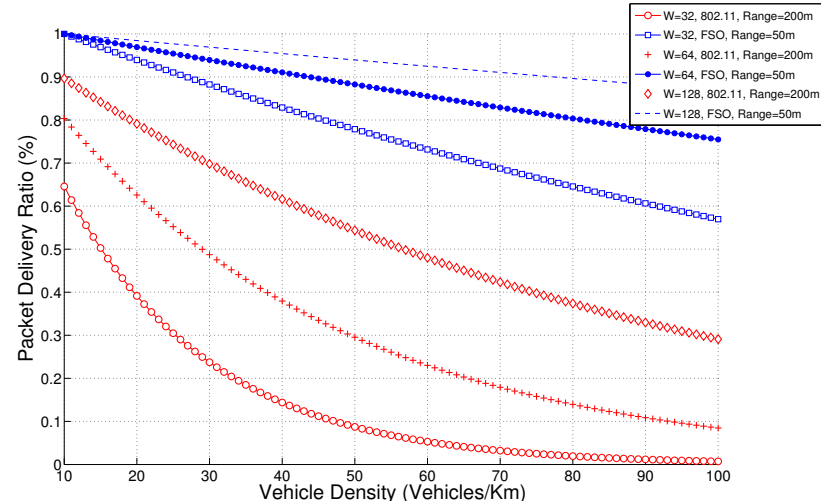

(b)

Figure 4: Analytical results from IEEE 802.11 and FSO techniques at various vehicle densities are shown. (a) shows how the number of nodes within the neighborhood of a vehicle. (b) shows the packet delivery ratio.

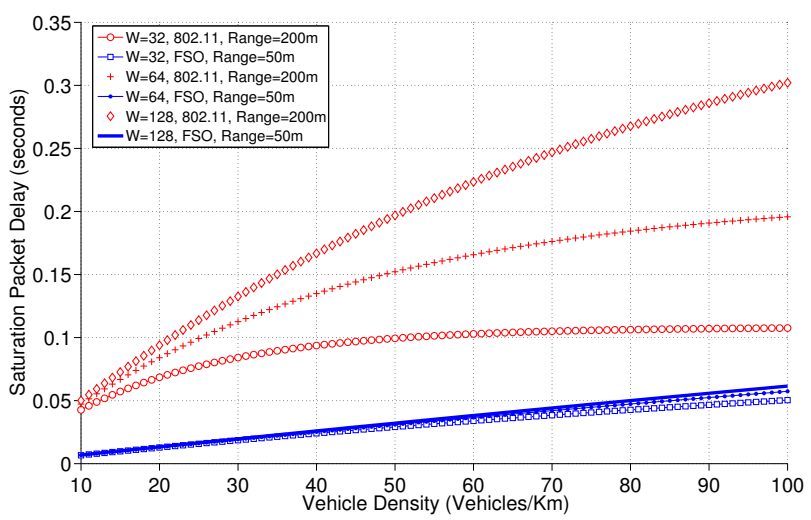

(a)

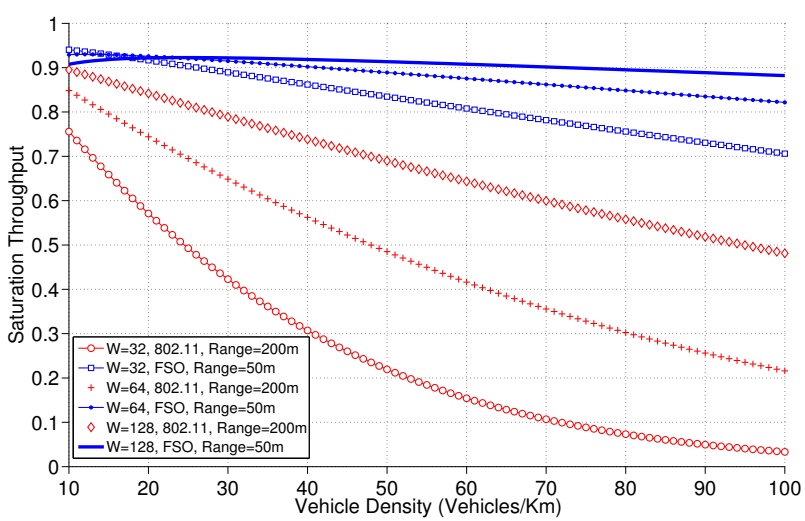

(b)

Figure 5: Simulation Results for IEEE 802.11 (range=200 m) and FSO (range=50 m) systems, showing (a) the delay per packet in seconds and (b) the normalized throughput (or utilization). 


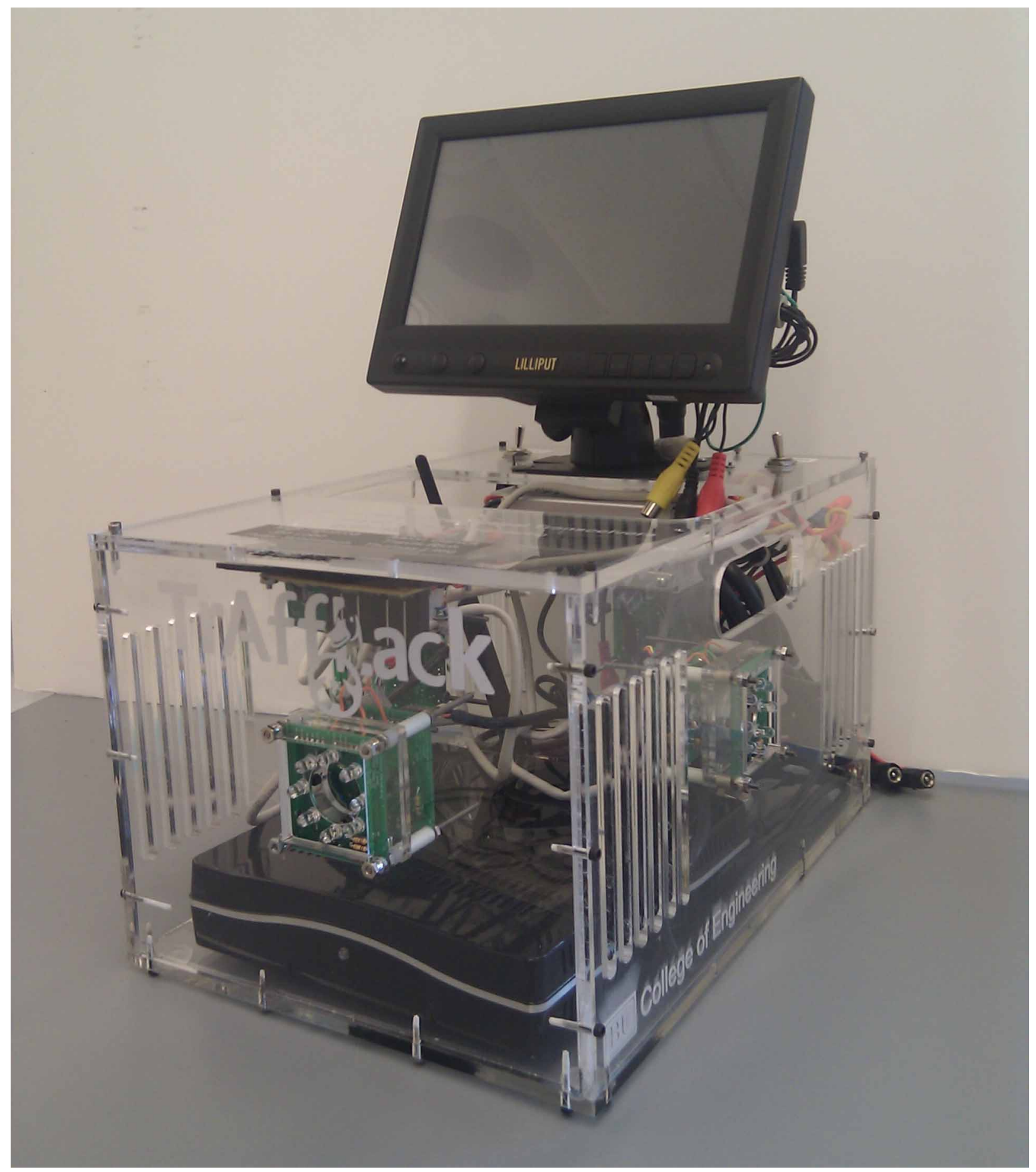

Figure 6: Shown is a photograph of the prototype of the vehicular communication system. The clear box serves as a mock car chassis, with the front and a side transceiver shown. Above the box is the touch-screen; inside the box are the car-computer and supporting electronics. 


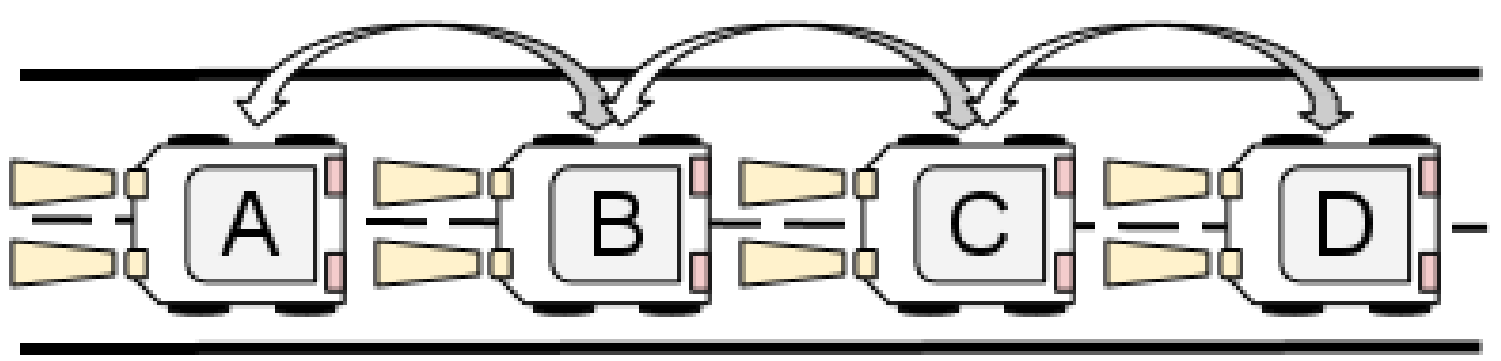

Figure 7: A scenario of multihop networking is depicted. In this scenario, headlight and taillight transceivers allow each vehicle to communicate with its nearest neighbor, but for vehicle $\mathrm{A}$ to communicate with vehicle $\mathrm{D}$, cooperation from intermediaries $\mathrm{B}$ and $\mathrm{C}$ is needed.

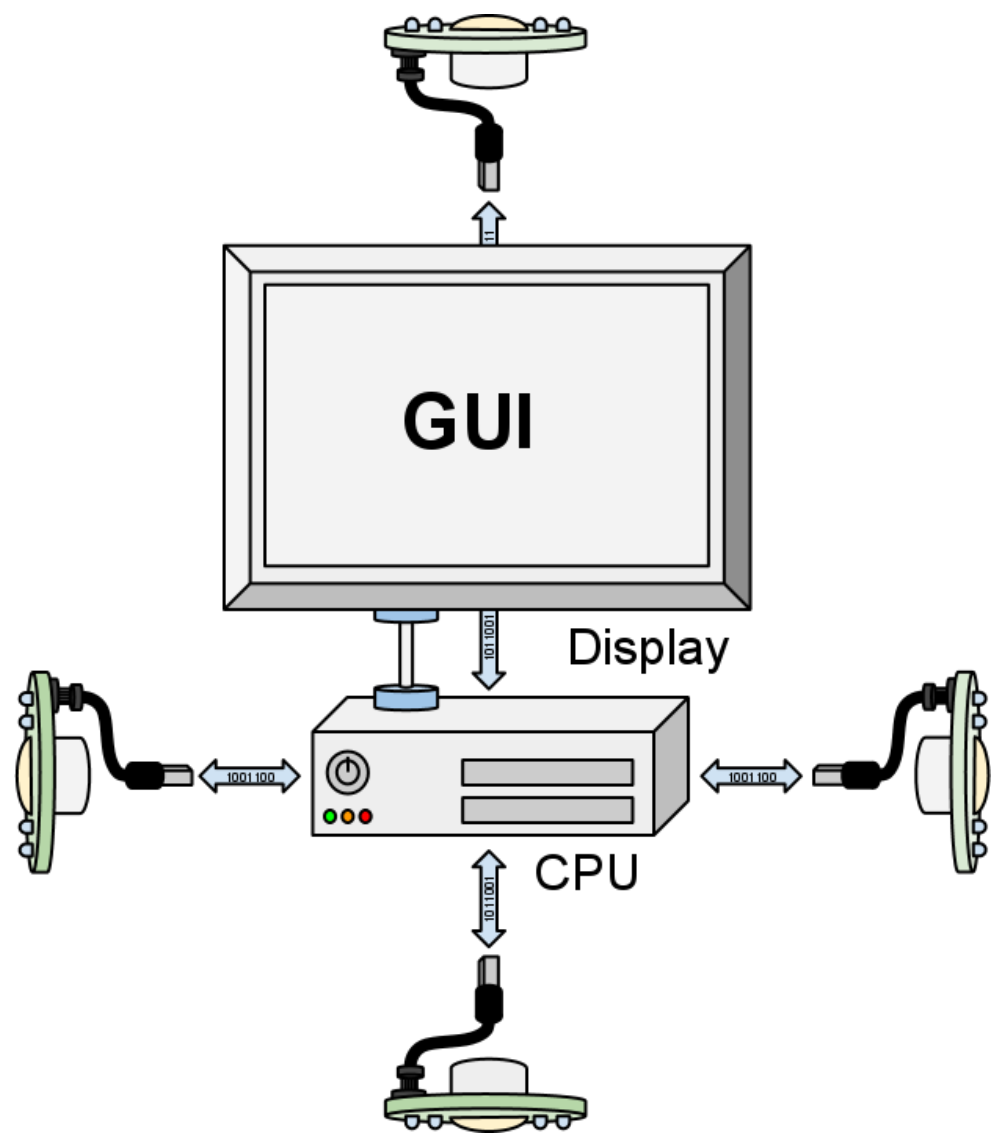

Figure 8: The connections between the car computer, the touch-screen, and the optical transceivers (facing forward, left, right, and backwards) are shown in this block diagram. 


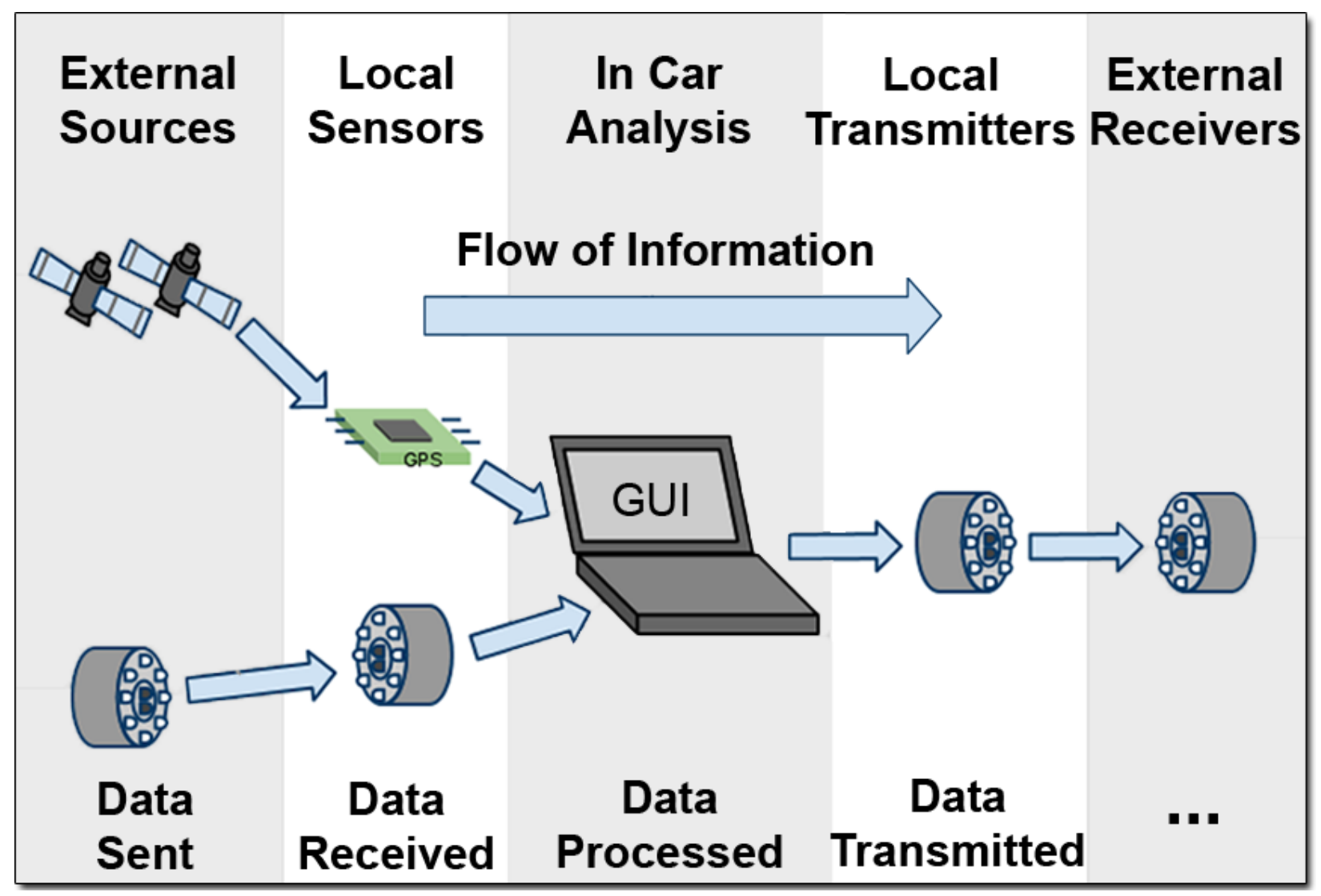

Figure 9: The flow of information through the system is shown in this flow-chart.

\begin{tabular}{|l|l|c|l|l|l|l|l|}
\hline \multicolumn{2}{|c|}{32} & 40 & 72 & 104 & 136 & 152 & 160 \\
\hline Preamble & ID & $\begin{array}{c}\text { Time \& } \\
\text { Date }\end{array}$ & Longitude & Latitude & Speed & Bearing & $\begin{array}{c}\text { Empty Bits for } \\
\text { Future Applications }\end{array}$ \\
\hline
\end{tabular}

Figure 10: The packets used by the prototype for V2V communications contain the fields shown. 


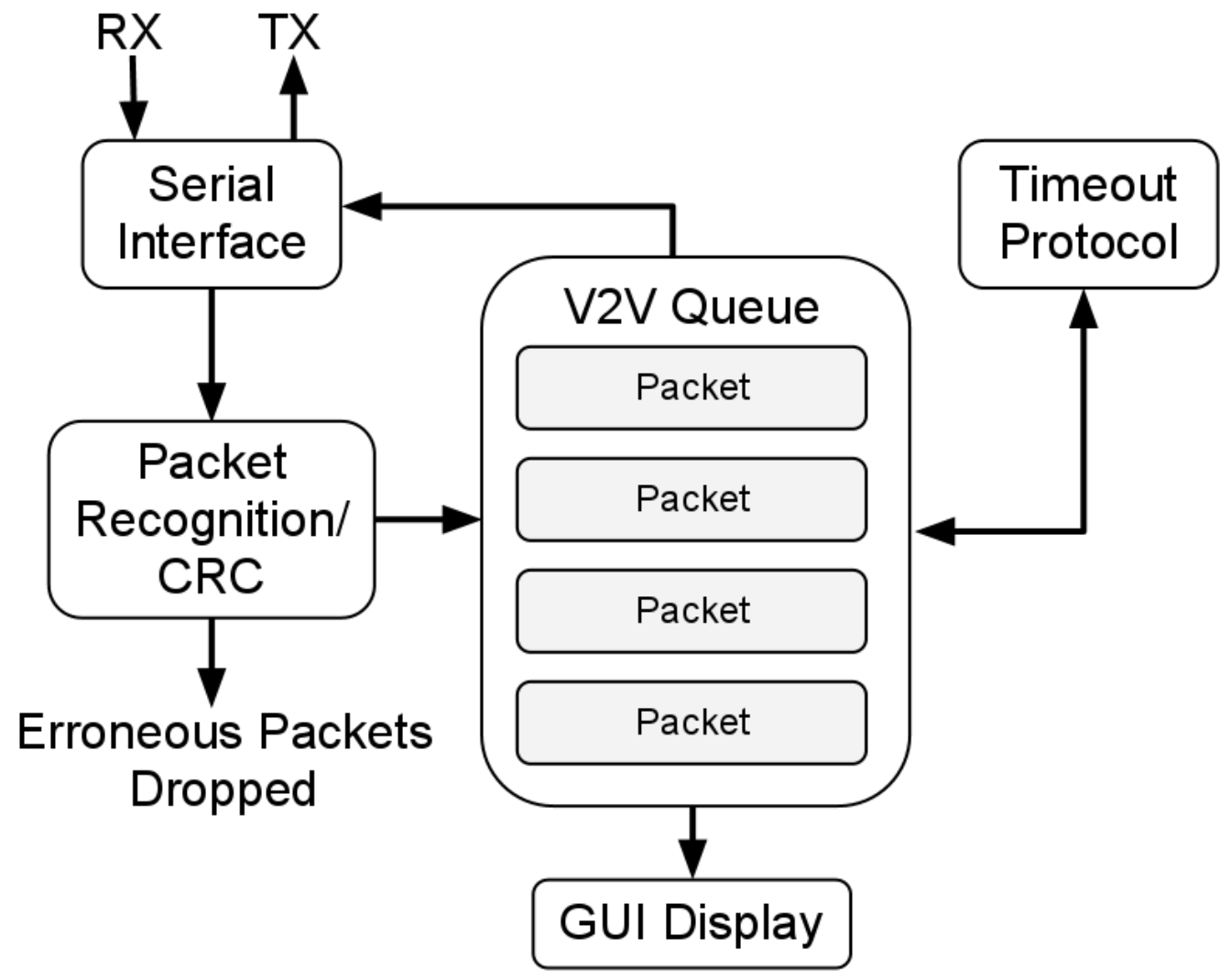

Figure 11: Received packets are verified and stored in a queue, where they can either expire, be retransmitted to neighboring vehicles, or presented to the user. 


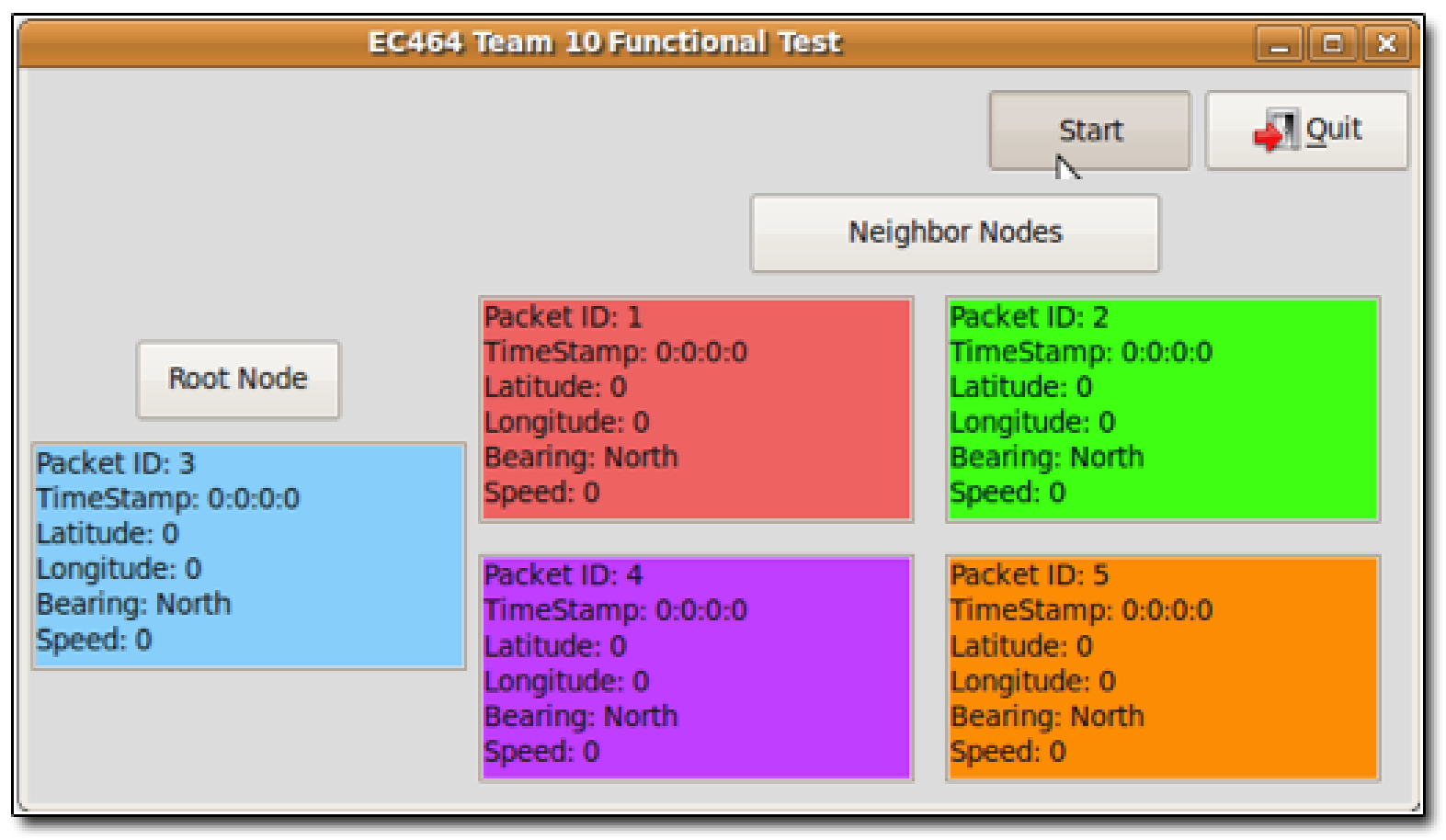

Figure 12: The current user interface presents the states of the user's own vehicle (shown under the "Root Node" heading) and the states of adjacent vehicles (shown under the "Neighboring Nodes" heading). 


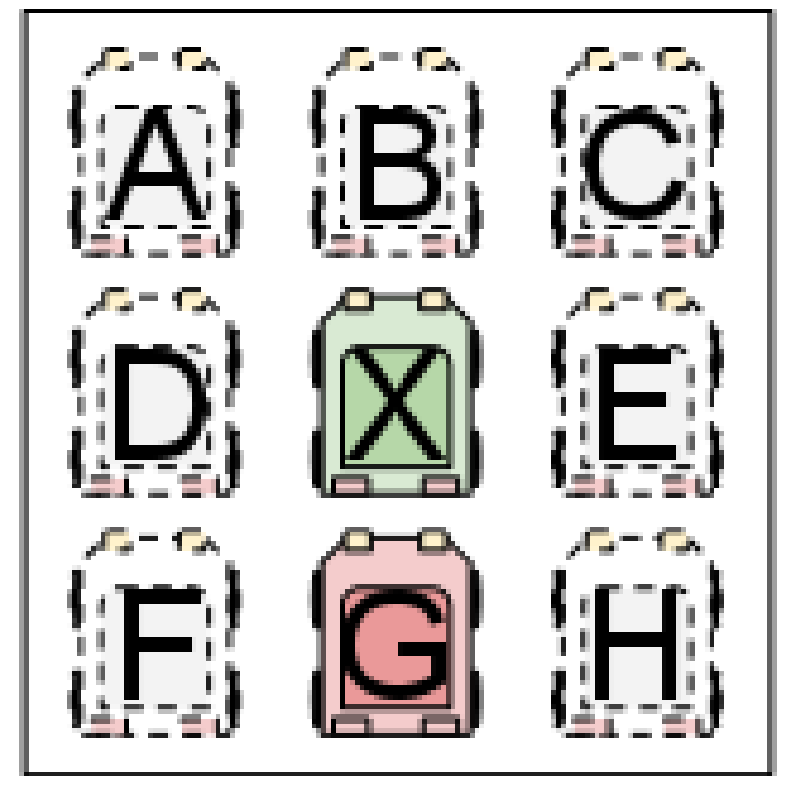

(a)

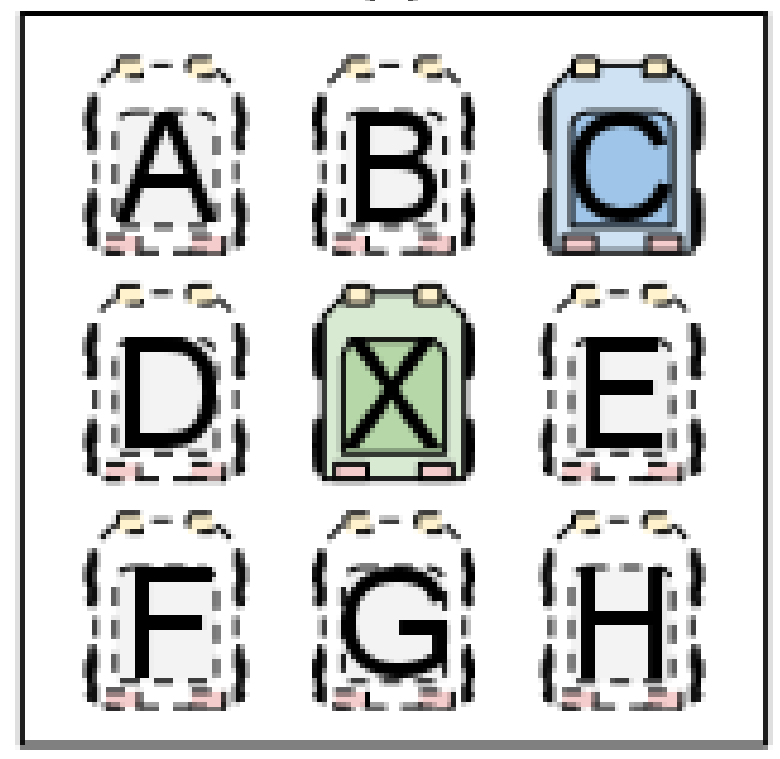

(c)

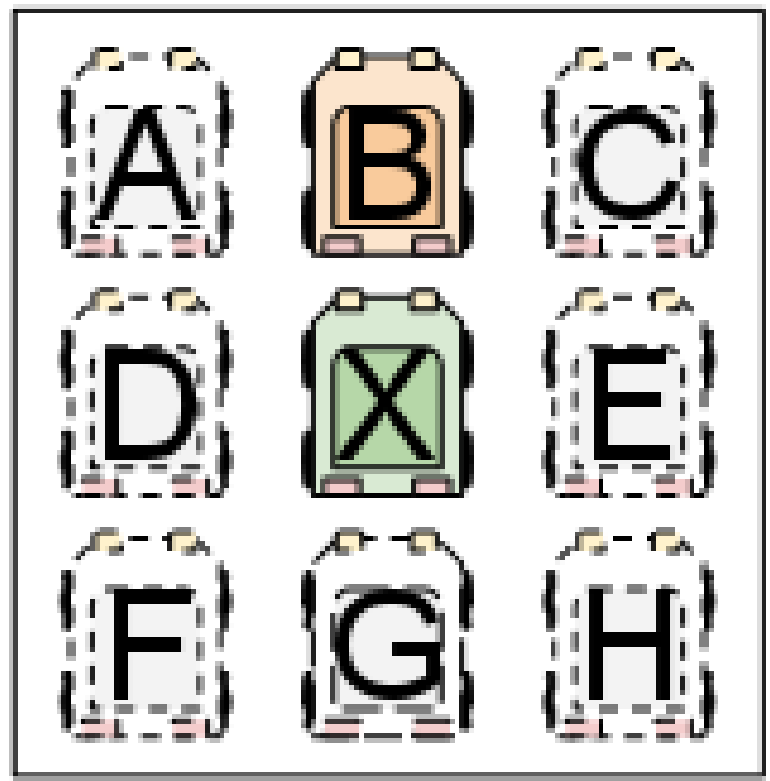

(b)

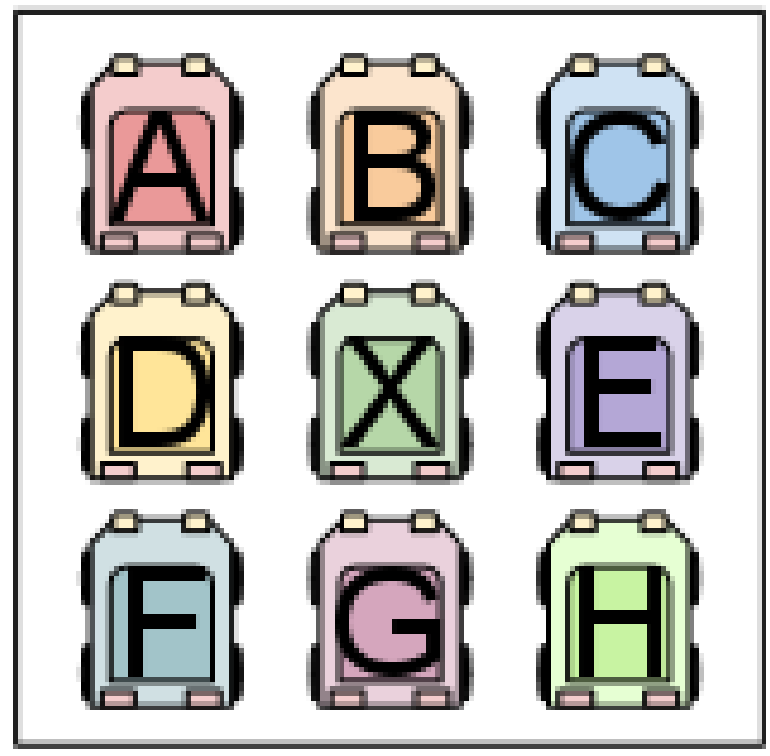

(d)

Figure 13: The new user interface improves situational awareness by graphically indicating the position of nearby vehicles. Colored vehicle icons indicate the presence of a vehicle at the depicted location. 